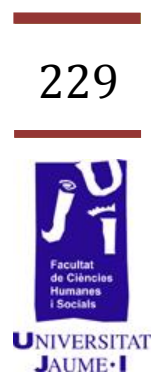

\title{
Inteligencia Emocional y Bienestar Subjetivo: un estudio en adolescentes
}

Veronica Andrés Cruz

veronicaandrescruz@gmail.com 
I. Resumen

En el presente estudio se pretende conocer la relación existente entre la Inteligencia Emocional (IE) y el Bienestar Subjetivo (BS), para saber si los adolescentes con mayor Inteligencia Emocional muestran un mayor Bienestar Subjetivo. Para ello, se ha aplicado el test MSCEIT para medir la IE y el test EBP para medir el Bienestar Subjetivo, estos han sido aplicados a 40 alumnos/as que cursan primer curso de Bachillerato. Los análisis estadísticos utilizados para dar respuesta a las hipótesis planteadas, han sido: descriptivos de las escalas para ambos test, comparación de medias y análisis correlacional entre ambos test. Además, plasmamos las diferencias entre género para ambos test. Los resultados obtenidos, en relación al objetivo planteado, nos muestran que no existe una clara influencia entre el nivel de IE y el BS. Otro aspecto importante obtenido es el análisis del BS y la IE por separado. En el caso del BS, vemos como es bajo para nuestros participantes. Esto se debe a la etapa de incertidumbre en la que se encuentran ya que, en esta etapa aparecen las primeras dudas en cuanto a su futuro académico y laboral, lo que puede desencadenar en estados de rumiación, baja autoestima, y como consecuencia puede producir un bajo bienestar. Por el contrario, en la IE obtenemos altas puntuaciones para su edad, hecho positivo para el alumnado. Estos resultados pueden ser de gran importancia para el ámbito educativo, ya que con frecuencia se ponen en práctica programas para fomentar el bienestar y la IE, en los que no se obtienen los resultados esperados.

Palabras clave: Inteligencia Emocional, Bienestar Subjetivo, adolescentes, género, MSCEIT, EBP.

\section{Introducción}

El objetivo principal de este trabajo de investigación ha sido conocer la relación existente entre la Inteligencia Emocional y el Bienestar Subjetivo. En el caso de la IE sería importante que el lector conociera los beneficios que aporta. Así, la IE aporta benceficios a la salud de los adolescentes a nivel emocional (López-Zafra y Jiménez, 2009). Tiene carácter protector en las conductas de riesgo, les ayuda a vencer las presiones del grupo de referencia para evitar el consumo y la adicción a las drogas (Trinidad y Johnson, 2002), la adquisición de la IE en la educación infantil tiene efecto preventivo de conductas desadaptativas en su futuro como adolescentes (Vallés, 2011), la baja IE puede ser un factor de la aparición del bullying (León, 2009) y los comportamientos delictivos (Extremera y Fernández-Berrocal, 2002). En el ámbito de las relaciones interpersonales, se ha visto como puede favorecer a que se tengan buenas relaciones interpersonales y mayores apoyos sociales (Ciarrochi y otros, 2001). 
Este trabajo pretende dar a conocer la situación real en cuanto a la fecilidad y al estado emocional de los adolescentes. Para así, dar pistas a los profesionales de la educación a la hora de adecuar programas emocionales. Puede ser que este tema parezca abstracto o algo irreal, pero creemos que este artículo puede dar muchas pistas a la hora de tratar con el alumnado que en breve deberá elegir su futuro y se enfrenta a una de las etapas que puede marcar su futuro profesional. Los estudiantes a los que hacemos referencia, son los que cursan primer curso de Bachillerato. En esta etapa aparecen las primeras dudas en cuanto a su futuro académico y laboral, lo que puede desencadenar en estados de rumiación, baja autoestima, lo que puede producir un bajo bienestar. Creemos que estos aspectos emocionales deben ser tenidos en cuenta a la hora de trabajar con el alumnado y crear un ambite positivo que fomente el bienestar.

También vemos como se han realizado muchos trabajos sobre IE y BS, pero estos han tratado los dos temas por separado o se ha evaluado un aspecto, y no se han centrado en nuestro grupo de edad, 17-19 años. Además, pretendemos que este trabajo sea de ayuda en el ámbito educativo. Ya que se ha visto que, si los programas de educación emocional y de prevención de conductas disruptivas están poco coordinados por el profesorado, no son evaluados, ni se lleva ningún control sobre su efecto en el alumnado, su impacto es nulo sobre la conducta del estudiantado y es poco probable que sus efectos se mantenga en el futuro (Greenberg y otros 2003).

\section{Marco Teórico}

Consideramos imprescindible definir los dos términos en los que se basa nuestro estudio. En el caso de la IE encontramos dos modelos principales, que la definen, estos son:

Modelo de rasgos o mixtos: concibe la IE como un conjunto de rasgos estables de personalidad, competencias socio-emocionales, aspectos motivacionales y habilidades cognitivas (Goleman, 1995). La más conocida de las propuestas y con la que se populariza el término es la de Goleman (1995), pese a haber tenido mucho éxito, este modelo carece de rigor científico y se basa en rasgos de personalidad positivos. (Extremera y Fernández-Berrocal, 2009).

Modelo de habilidad: es el propuesto por Salovey y Mayer, los cuales interpretan la IE como una inteligencia basada en el uso de las emociones de forma adaptativa y su aplicación a nuestro pensamiento (FernándezBerrocal y Extremera, 2009). No conciben la IE como un rasgo de personalidad, sino como una habilidad cognitiva que facilitan la adaptación de nuestras emociones al medio, para tener mayor bienestar (Vallés, 2007). Este modelo concibe la IE como el dominio de cuatro habilidades emocionales: 1) Percepción, evaluación y expresión 
emocional; 2)Facilitación emocional;3) Comprensión emocional y 4)Regulación emocional. En el presente estudio, nos hemos basado en el modelo de habilidad propuesto por Mayer y Salovey porque ha mostrado mayor validez que los otros modelos, su planteamiento es el que mejor relación ha revelado con otros tipos de inteligencias.

El término Bienestar Subjetivo o hedónico se encuadra en el marco teórico de la Psicología Positiva (Seligman, 1998), la cual se centra en aspectos tales como las experiencias positivas, los rasgos individuales positivos (fortalezas y virtudes), sus efectos y las instituciones que facilitan su desarrollo. se relaciona con la consecución de estados afectivos positivos, la evitación de los negativos y la satisfacción vital.

Si nos planteamos la relación que tienen estos dos conceptos de estudio, encontramos evidencias empíricas que los relacionan. Así, se ha demostrado que niveles bajos de IE pueden llevar a una alta sintomatología depresiva, ya que los sujetos con alta IE son capaces de disminuir la intensidad y frecuencia de los estados de ánimo negativos provocados por acontecimientos cotidianos (Extremera y FernándezBerrocal, 2006). Por el contrario, una elevada IE, está asociada con indicadores positivos de bienestar y ajuste psicológico, mayor satisfacción vital (Extremera, y otros (eds.) 2009) como una alta autoestima (Echevarría y López-Zafra, 2011; Rey, y otros, 2011), una satisfacción vital mayor (Extremera, Durán y Rey, 2009) y mayores niveles de felicidad subjetiva y bienestar (Augusto y otros, 2011; Extremera y otros, 2011). Además, la capacidad, para atender a los estados afectivos, reconocer los propios sentimientos y reparar las emociones es necesaria para un buen funcionamiento, son esenciales para el afrontamiento adaptativo al estrés y la mejora del bienestar emocional (Salovey, y otros, 1999).

\section{Objetivos}

El objetivo principal que nos planteamos es conocer la relación existente entre la IE y el Bienestar Subjetivo. Como objetivos específicos nos planteamos:

- Estudiar la relación existente entre la IE y el Bienestar Subjetivo, para los sujetos que componen nuestra muestra.

- Comprobar si las escalas del test MSCEIT, correlacionan significativamente con las escalas de bienestar subjetivo y material del cuestionario EBP (Escala de Bienestar Psicológico).

- Comprobar si existen diferencias significativas entre sexos en cuanto a la IE y al BS. 


\section{Hipótesis}

Las hipótesis que nos planteamos en la presente investigación son las siguientes:

- H1: Existe una correlación positiva entre el nivel de IE y el nivel de Bienestar Subjetivo.

- H2: Los hombres obtendrán un mayor nivel de Bienestar Subjetivo que las mujeres.

○ H3: Se obtendrán puntuaciones más altas de IE en mujeres.

\section{Material y método}

\section{a) Participantes y procedimiento}

Debido a nuestro interés de conocer la IE y el BS en la población adolescente. Además, teniendo en cuenta las variables de estudio, decidimos tomar datos de una muestra compuesta por 40 alumnos de primer curso de bachillerato, de los cuales el 14 (36,6\%) son hombres y 26 $(63,4 \%)$ mujeres. Su rango de edad es de 17 a 18 años.

Para realizar la presente investigación seguimos los siguientes pasos: Se habló con la orientadora sobre el trabajo a realizar para que lo comunicara al equipo directivo y a los padres del alumnado. La aplicación de los cuestionarios se realizó en una sola sesión y en grupo, durante la sesión de tutoría. Al alumnado se le indicó que el objetivo del estudio era el conocimiento de su situación de su estado emocional y su bienestar subjetivo, se indicó que las respuestas eran de carácter anónimo y confidencial. Durante la sesión, se hicieron las aclaraciones oportunas cuando los participantes presentaban dudas. Tras la recogida de información, se procedió a la corrección de los cuestionarios. En el caso del test MSCEIT, ésta se ha realizado a través de Internet. En el caso del test EBP, la puntuación se obtuvo de forma manual, sumando las puntuaciones obtenidas. El análisis de las puntuaciones obtenidas se hizo mediante el paquete estadístico SPSS.

\section{b) Instrumentos}

Para evaluar la Inteligencia Emocional se utilizó la adaptación española de Natalio Extremera y Pablo Fernández-Berrocal del test de Inteligencia Emocional MSCEIT (2009). (Test de Inteligencia Emocional Mayer-SaloveyCaruso). Esta prueba ha sido elegida porque muestra una fiabilidad alta y es una prueba validada. EI MSCEIT está compuesto por 141 ítems y está 
diseñado para medir las cuatro áreas principales de la inteligencia emocional, según el modelo de Mayer y Salovey.Se evaluó el nivel de Bienestar Subjetivo de los adolescentes mediante la Escala de Bienestar Psicológico (EBP) de José Sánchez-Cánovas (1998). Esta prueba ha sido elegida porque muestra una fiabilidad alta y es una prueba validada. Está formada por 65 ítems, con valoraciones que van desde 1 a 5 . Esta prueba consta de cuatro subescalas: 1) subescala de bienestar psicológico subjetivo; 2) subescala de bienestar material; 3)subescala de bienestar laboral y 4 )subescala de relaciones con la pareja. De acuerdo con la muestra y el objetivo de nuestro estudio, aplicamos las escalas de Bienestar psicológico subjetivo y de Bienestar material.

\section{Resultados}

\section{a) Escala de Bienestar Subjetivo (EBP): Análisis descriptivos}

A continuación, en la Tabla 1 se muestra el resumen de casos del total de los participantes, la muestra se ha dividido por género, mostrando las puntuación Media, el nivel de significación, el número de sujetos, la puntuación T y la Desviación Típica (DT) obtenida de la Escala Bienestar Psicológico (EBP). Para conocer las diferencias significativas entre sexos, hemos realizado una prueba $T$ para muestras independientes, tras realizar este análisis estadístico obtenemos la significación que es el valor que nos determinará si existen diferencias entre sexos.

Tabla 1. Medias, DT y prueba T para muestras independientes

\begin{tabular}{|c|c|c|c|c|c|c|}
\hline & Género & $\mathbf{N}$ & Media & DT & $\mathbf{T}$ & Sig. \\
\hline \multirow{2}{*}{$\begin{array}{l}\text { Bienestar } \\
\text { Subjetivo }\end{array}$} & Hombre & 14 & 114,6429 & 11,01473 & \multirow{2}{*}{ - , 170 } & \multirow{2}{*}{,866 } \\
\hline & Mujer & 26 & 115,4615 & 16,04551 & & \\
\hline Bienestar & Hombre & 14 & 37,2857 & 6,24412 & \multirow{2}{*}{$-1,201$} & \multirow{2}{*}{ 237 } \\
\hline Material & Mujer & 26 & 39,8462 & 6,52805 & & \\
\hline \multirow{2}{*}{ Total } & Hombre & 14 & 151,9286 & 15,59321 & \multirow{2}{*}{,- 333} & \multirow{2}{*}{, 741} \\
\hline & Mujer & 26 & 154,0000 & 20,20495 & & \\
\hline
\end{tabular}

En el caso de la Escala de Bienestar Subjetivo no encontramos diferencias significativas $(\alpha=.866, p .>.05)$. En el caso de la escala de Bienestar Material no se encuentran diferencias significativas $(\alpha=.237,>.05)$, las medias de hombres y mujeres no difieren. En cuanto a la puntuación total, que resulta de la suma de las escalas anteriormente explicadas tampoco se obtienen diferencias significativas ( $\alpha=.741, p>.05)$, las $M$ para 
ambos sexos son muy parecidas no difieren en cuanto a su puntuación. Los resultados no muestran diferencias estadísticamente significativas en lo que respecta al género.

En el planteamiento de la investigación, esperábamos obtener que la muestra de hombres obtenga mayores puntuaciones en esta prueba porque tiene un mejor autoconcepto y se valoran a sí mismos de una forma más positiva, como muestra Rodríguez. A (2005), en su investigación realizada con una muestra parecida en cuanto rango de edad. En las investigaciones realizadas por Lucero y Cornejo (2005); y Fernández, C. y Polo, M. T. (2010) también muestran puntuaciones de bienestar más bajas las mujeres, como resultado de mayores preocupaciones.

\section{b) Resultados del test MSCEIT: Análisis descriptivos}

A continuación, en la Tabla $\mathbf{2}$ se muestra el resumen de casos del total de los participantes divididos por género; se muestra la puntuación Media, el nivel de significación, el número de sujetos, la puntuación T y la Desviación Típica (DT) obtenida en el test MSCEIT para cada una de las áreas y ramas. Para conocer las diferencias significativas entre sexos, hemos realizado una prueba $\mathrm{T}$ para muestras independientes, tras realizar este análisis estadístico obtenemos la significación que es el valor que nos determinará si existen diferencias entre sexos.

Tabla 2. Medias, DT y prueba T para muestras independientes

\begin{tabular}{|c|c|c|c|c|c|c|}
\hline & Género & $N$ & Media & DT & T & Sig. \\
\hline \multirow[b]{2}{*}{ CIE } & Hombre & 14 & 98,4286 & 11,07814 & \multirow{2}{*}{,- 680} & \multirow{2}{*}{,501 } \\
\hline & Mujer & 26 & 101,3846 & 14,06009 & & \\
\hline \multirow[b]{2}{*}{ CIEX } & Hombre & 14 & 103,7143 & 16,26430 & \multirow{2}{*}{1,064} & \multirow{2}{*}{ 294 } \\
\hline & Mujer & 26 & 99,0000 & 11,57929 & & \\
\hline \multirow[b]{2}{*}{ CIES } & Hombre & 14 & 95,9286 & 9,03382 & \multirow{2}{*}{$-1,633$} & \multirow{2}{*}{ 111 } \\
\hline & Mujer & 26 & 104,0385 & 17,28926 & & \\
\hline \multirow[b]{2}{*}{ CIEP } & Hombre & 14 & 106,2857 & 17,73058 & \multirow{2}{*}{ 698 } & \multirow{2}{*}{,489 } \\
\hline & Mujer & 26 & 103,1154 & 11,03568 & & \\
\hline \multirow[b]{2}{*}{ CIEF } & Hombre & 14 & 98,3571 & 9,11978 & \multirow{2}{*}{1,241} & \multirow{2}{*}{ 222 } \\
\hline & Mujer & 26 & 94,1154 & 10,87870 & & \\
\hline
\end{tabular}




\begin{tabular}{|c|c|c|c|c|c|c|}
\hline \multirow{2}{*}{ CIEC } & Hombre & 14 & 95,2143 & 8,22920 & $-1,192$ & \multirow{2}{*}{, 241} \\
\cline { 2 - 5 } & Mujer & 26 & 100,6154 & 15,77359 & & \\
\hline \multirow{2}{*}{ CIEM } & Hombre & 14 & 96,7857 & 10,47472 & $-1,775$ & \multirow{2}{*}{, 084} \\
\cline { 2 - 5 } & Mujer & 26 & 105,7308 & 17,14773 & & \\
\hline
\end{tabular}

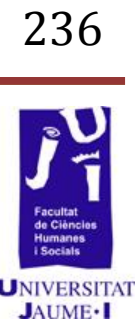

Las mujeres han obtenido una puntuación media mayor en la escala total (CIE), aunque las diferencias son mínimas, tras ver los niveles de significación para esta escala, vemos como no se obtienen diferencias significativas entre hombres y mujeres $(\alpha=.501, p>.05)$. Por lo que, no podemos decir que la puntuación de las mujeres difiere de la de los hombres. En el área experiencial (CIEX) los hombres obtienen una puntuación media de 103.71 y una DT de 16.26; a diferencia de las mujeres que obtienen una puntuación media de 99.00 puntos y una DT de 11,58; pese a ser una diferencia muy pequeña, esto nos puede indicar que en nuestra muestra los hombres tiene mayor capacidad para comparar la información emocional con otros tipos de experiencias sensoriales como pueden ser los colores o sonidos. Tras el análisis de significación vemos que las diferencias que se obtienen entre sexos no son significativas ( $\alpha=.294, p>.05$ ). En cuanto al área estratégica (CIES), los hombres obtiene una puntuación $M$ de 95,93 y una DT de 9,03 y las mujeres obtienen una puntuación M de 104,04 y una DT de 17,29. Las diferencias entre medias son muy pequeñas, vemos el análisis de significación( $\alpha=.111, p>.05)$, tras este análisis interpretamos que no existen diferencias entre sexos.

Tras haber analizado las puntuaciones de área obtenidas pasaremos a las puntuaciones de rama. Así, empezaremos con la rama de Percepción Emocional (CIEP), las puntuaciones obtenidas por los hombres han sido superiores Pero la diferencia con las mujeres es muy poca, tal y como nos indica la media. En este caso los hombres tienen mayor habilidad para prestar atención y decodificar las señales emocionales del tono de voz, expresiones artísticas y faciales de los sujetos. Aunque, estos datos no son significativos, ya que el análisis de significación no nos muestra diferencias significativas entre sexos $(\alpha=.489, p>.05)$.

En el caso de la facilitación emocional (CIEF), los hombres han obtenido puntaciones mayores en muy pocos puntos, en este caso las mujeres han obtenido una puntuación media de 94,12 y los hombres de 98,36. Así, los hombres tienen mayor capacidad para utilizar las emociones para solucionar problemas, tomar decisiones, concentrarse, razonar de forma efectiva. Como señalábamos, las medias no difieren y tras el análisis de significación comprobamos que no existen diferencias significativas entre $\operatorname{sexos}(\alpha=.222, p>.05)$. 
En cuanto a la rama de Comprensión emocional (CIEC), las mujeres obtienen puntuaciones mayores a los hombres, lo que nos muestra que las mujeres tienen mayor capacidad de etiquetar las emociones de forma más eficaz; conocen en que tipo de emociones pueden desencadenar las situaciones. Tras el análisis de significación vemos que las diferencias que se obtienen entre sexos no son significativas ( $\alpha=.241, p>.05)$.

Por último, en la rama de Manejo emocional (CIEM), las mujeres puntúan más alto que los hombres; con una puntuación media de 105,73 puntos y los hombres con 96,79 puntos. Esta puntuación es la que muestra mayor diferencia de las obtenidas en las ramas y en las tareas. Lo que muestra que, las mujeres tendrán mayor capacidad para manejar las emociones de forma óptima, serán capaces de utilizar sus sentimientos para tomar mejores decisiones y no reprimiran sus sentimientos. Para asegurarnos de que existan diferencias significativas entre sexos miramos el nivel de significación que hemos obtenido, vemos que no hay diferencias significativas entre sexos $(\alpha=.084, p>.05)$.

\section{c) Relaciones entre el test MSCEIT y la escala EBP: Análisis correlacionales}

Hemos realizado un análisis de correlación de Pearson para comprobar si los sujetos que obtengan mayores puntuaciones en el test MSCEIT también mostrarán unas mayores puntuaciones en el test de bienestar EBP. Se han obviado las variables, sexo y edad, ya que la edad de los sujetos no varía y en cuanto a sexos la muestra de hombres es inferior a la de las mujeres.

Tabla 3. Correlaciones test MSCEIT y EBP

\begin{tabular}{|c|c|c|c|c|c|c|c|c|c|c|c|c|c|c|c|}
\hline & Cie & Ciex & Cies & Ciep & Cief & Ciec & Ciem & Cara & dibu & Faci & Sens & Cam & Com & man & emo \\
\hline B. Sub. & 119 & 120 & 142 & 105 & ,067 & 151 & ,069 & -,193 & 216 &,- 042 & 169 &,- 006 & 254 & ,014 & 126 \\
\hline B.Mate. & 281 & 201 & ,327* & 251 & ,067 & 262 & 295 &,- 049 & ,349* &,- 192 & 292 & 164 & 261 & 199 & ,374* \\
\hline Total & 224 & 221 & ,214 & 220 & 136 & 184 & 161 &,- 161 & ,342* &,- 050 & 263 & ,050 & 242 & ,061 & 265 \\
\hline
\end{tabular}

Tras analizar los resultados, obtenemos que la subescala de bienestar material correlaciona con el área estratégica $(r=.327, p<.05)$. Además, muestra relaciones significativas con la tarea dibujos $(r=.349, p<.05)$, esta tarea se encuentra dentro de la rama de percepción emocional; la escala de bienestar material también muestra relaciones significativas con la 
tarea relaciones emocionales $(r=.374, p<.05)$, esta tarea forma parte de la rama manejo de emociones. La puntuación total que comprende la suma total de las subescalas bienestar subjetivo y bienestar material correlaciona con la tarea dibujos $(r=.342, p<.05)$.

Los resultados obtenidos tras el análisis correlacional no nos muestran una relación desde el ámbito emocional como se esperaba, ya que las puntuaciones obtenidas en la escala de Bienestar Subjetivo no han correlacionado con ninguna puntuación obtenida por el alumnado en Inteligencia Emocional.

\section{Discusión y conclusiones}

El objetivo del estudio es conocer si el alumnado con alta puntuación en Inteligencia Emocional también muestra un mayor nivel de bienestar subjetivo. A continuación, se muestran la discusión de los resultados para cada hipótesis planteada:

La primera hipótesis tenía como objetivo demostrar que existe una correlación positiva entre el nivel de IE y el Bienestar subjetivo (Brackett y Mayer, 2003; Brackett et al., 2006) esta hipótesis no se da en nuestro estudio, esto podría ser debido a que tenemos una muestra pequeña en comparación a los estudios realizados por estos autores. Tras el análisis de correlación, vemos como las escalas de Bienestar Material y Subjetivo del test EBP no correlacionan con las ramas del test MSCEIT. Pese a esto, encontramos que la escala de Bienestar Material correlaciona con el área estratégica, la tarea dibujos y las relaciones emocionales. También encontramos correlación positiva entre la escala total del test EBP con la tarea dibujos del test MSCEIT.

Es importante tener estos aspectos en cuenta, el área estratégica evalúa la capacidad del sujeto para comprender y manejar las emociones sin percibir ni experimentar necesariamente los sentimientos correctamente. Indica la precisión con que el sujeto comprende el significado de las emociones y cómo puede manejares las propias emociones y las de los demás. La tarea dibujos evalúa la capacidad de percibir emociones a través del arte. La tarea relaciones emocionales forma parte de la escala manejo emocional de test, esta tarea concreta evalúa la capacidad del sujeto para incorporar emociones en el proceso de toma de decisiones que implican a otras personas. Por ello, que los participantes puntúen alto en bienestar material puede significar que los sujetos tengan una buena capacidad para comprender y manejar sus emociones, tengan una buena precisión para comprender el significado de sus propias emociones y el de los demás. Además, pueden ser buenos percibiendo emociones en manifestaciones artísticas y que tengan una buena capacidad de toma de decisiones cuándo éstas impliquen a otros sujetos. 
La segunda hipótesis planteada es que los hombres obtendrán un mayor nivel de Bienestar Subjetivo que las mujeres, tal y como mostraba la investigación realizada por Rodríguez (2005) en una muestra muy parecida a la nuestra. En su investigación, atribuía un mejor Bienestar Subjetivo a los hombres, debido a un mejor autoconcepto físico positivo, lo que aporta a los hombres mayor seguridad. Nuestros resultados no van en esta línea, esto puede ser debido a que nuestra muestra es muy inferior a la utilizada por Rodríguez (2005), en su caso fue de 540 estudiantes. Por otra parte, Cornejo y Lucero (2005) y Fernández, C. y Polo, M. T. (2011), también mostraban unas puntuaciones inferiores en el caso de las mujeres para el Bienestar Subjetivo, en este caso, las puntuaciones bajas por parte de las mujeres eran atribuidas a que muestran mayores preocupaciones.

La tercera hipótesis que nos planteamos en este estudio es que las mujeres obtendrán puntuaciones más altas que los hombres en el test MSCEIT. En nuestro estudio esta hipótesis no se cumple, las puntuaciones para hombres y mujeres no muestra diferencias significativas. En nuestro estudio vemos como tanto hombres como mujeres, se encuentran en un nivel competente de IE, lo que se traduce en una habilidad suficiente para desenvolverse con éxito para reconocer, percibir, comprender y regular sus emociones, las de las personas que les rodean y las que el ambiente les exigen para tener un comportamiento adaptativo. Los resultados obtenidos en esta hipótesis no irían en la línea de los obtenidos por Fernández-Berrocal y Extremera (2009), cuando validaron el test con la muestra española. Estos resultados estarían en la línea de los encontrados por Salvador y Morales (2009), estos autores no encontraron diferencias significativas en IE utilizando el test TMMS (instrumento de medida del modelo de habilidad de Mayer y Salovey), entre hombres y mujeres mexicanos con edades comprendidas entre los 22 y los 58 años.

Puede ser muy positivo que en nuestro estudio no se aparezcan diferencias entre sexo, en investigaciones anteriores se dan diferencias qué podrían ser debidas a la crianza diferenciada entre hombres y mujeres, criándose éstas en las competencias interpersonales, mayores capacidades afectivas, que en el caso de los hombres no se creen necesarias (Candela et, al. 2002). Estos autores, hacen un análisis sobre tres investigaciones en las qué no se encuentran diferencias significativa entre hombres y mujeres en IE.

Tras los resultados, vemos que en el caso de nuestros participantes se debería mejorar el aspecto del Bienestar subjetivo, ya que sus puntuaciones son medias. Nuestros participantes se encuentran en una etapa de incertidumbre, debido al nivel de estudios en el qué se encuentra, ya que están cursando primer curso de bachillerato y en esta etapa aparecen las primeras dudas en cuanto a su futuro académico y laboral, lo que puede desencadenar en estados de rumiación, baja 
autoestima, lo que puede producir un bajo bienestar. Por ello, sería interesante adecuar el programa de Palomera, R. (2009).

En el caso de la IE los participantes tienen puntuaciones altas para su edad, hecho positivo para el alumnado. Por ello, sería muy bueno que tras este estudio se dieran pistas al centro de cómo adaptar un material de educación emocional para el alumnado de bachillerato y de último ciclo de Educación Secundaria para que el bienestar del alumnado se incrementara, ya que en estos grupos de edad no se sigue ningún material específico. Se buscarían actividades para crear un ambiente de clase positivo, que incrementaran la autoestima, el afecto positivo, actividades reforzantes, tanto para el profesorado como para el alumnado. Unas pautas interesantes para el alumnado y profesorado serían las propuestas por Colom y Froufe (199), los cuales consideran importante plantear actividades, tanto para el alumnado como para el profesorado, estos autores creen que el alumnado imita a sus profesores/as.

\section{Bibliografía}

Augusto, J. M. y otros (eds.) (2011). Does Perceived Emotional Intelligence and Optimism/pessimism Predict Psychological Well-being? Journal of Happiness Studies, 12, 463-474.

BoYATZIS, R., y otros (eds.) (2000). Clustering competence in emotional intelligence: Insights from the emotional competence inventory (Eds.). En Bar-on, R. y Parker, J. D. A. (Eds.), Handbook of Emotional Intelligence (pp. 343-362). San Francisco: Jossey-Bass.

BRACKETT, M. A. y otros (eds.) (2003). Convergent, discriminant, and incremental validity of competing measures of emotional intelligence. Personality and Social Psychology Bulletin, 29, 1147-1158.

BRACKETT, M. y otros (eds.) (2006). Relating emotional abilities to social functioning: A comparison of self-report and performance measures of emotional intelligence. Journal of Personality and Social Psychology, 91, 780-795.

CAndela, A., y otros (eds.) (2002). Inteligencia Emocional y la variable género. Revista Electrónica de Motivación y Emoción, 5 (10). http://reme.uji.es/articulos/acandc2272105102/texto.html

CANESSA, B. (eds.) (2000). Adaptación española "Esscalas de afrontamiento para adolescentes" en escolares entre 14 y 17 años de edad de diferente sexo y nivel socioeconómico. Citada por Cornejo, M. y Lucero, M. C. (2005). Preocupaciones vitales en estudiantes universitarios relacionado 
con bienestar psicológico y modalidades de afrontamiento. Fundamentos en Humanidades, 2 (12), 143-153.

CiARROCHI, J. y otros (eds.) (2001). Measuring emotional intelligence in adolescents. Personality and Individual Differences, 31(7), 1105-1119.

Colom, R. y FroufE, M. (1999). Inteligencia emocional: cómo aplicarla en la práctica docente. Cuadernos de Educación. Madrid: Santillana

CoRnejo, M. y LUCERo, M. C. (2005). Preocupaciones vitales en estudiantes universitarios relacionado con bienestar psicológico y modalidades de afrontamiento. Fundamentos en Humanidades, 2 (12), 143-153.

EChevarRíA, A. y López-Zafra, E. (2011). Pigmalión, ¿Sigue vivo? Inteligencia emocional y la percepción del profesorado de alumnos de E.S.O. Boletín de Psicología, 102, 7-22.

Extremera, N. y Fernández-Berrocal, P. (2006). Emotional Intelligence as predictor of the mental, social, and physical health in university students. Spanish Journal of Psychology, 9, 45-51.

Extremera, N. y Fernández-Berrocal, P. (2009). Inteligencia emocional, afecto positivo y felicidad. En Fernández-Abascal, E (Eds.) Emociones positivas (pp.229-246). Psicología Pirámide.

Extremera, N. y otros (eds.) (2011). Trait Meta-Mood and Subjective Happiness: A 7-week Prospective Study. Journal of Happiness Studies, 12, 509-517.

Fernández-Berrocal, P. y Extremera, N. (2002). La inteligencia Emocional como una habilidad en la escuela. OEI- Revista Iberoamericana de Educación, 33 (8), 1-10.

FERnÁNDEZ-BERROCAL, P. y otros (eds.) (2009). ¿Es posible crear una escuela emocionalmente inteligente? En Augusto, J. M. (Ed.) Estudios en el ámbito de la inteligencia emocional (pp.37-55). Universidad de Jaén.

FernándeZ, C. y Polo, M. T. (2011). Afrontamiento, estrés y bienestar psicológico en estudiantes de Educación Social de nuevo ingreso. EduPsykhé, 10 (2), 177-192.

Goleman, D. (1995). Emotional Intelligence. New York: Bantam Books. (Trad. cast. Kairós, 1996).

GREENBER, M. T, y otros (eds.) (2003). Enhancing school-based prevention and Routh development through coordinated social, emocional, and academic learninig, American Psychologist, 58, 466-474. 
LEón, B. (2009). Salud mental en las aulas. Revista de Estudios de Juventud, 84, 66-83.

LÓPEZ-ZAFRA, E. y JIMÉNEZ, M. (2009). Inteligencia emocional y rendimiento escolar:estado actual de la cuestión. Revista Latinoamericana de Psicología, 41 (1), 69-79.

PAlOMERA, R. (2009). Educando para la felicidad. En Fernández-Abascal, E. (Eds.) Emociones positivas (pp. 254- 272). Psicología: Pirámide.

REY, L. y otros (eds.) (2011). Perceived Emotional Intelligence, Self-Esteem and Life Satisfaction in Adolescents. Psychosocial Intervention, 20, 227234.

ReY, L. y EXTREMERA, N. (2012). Inteligencia emocional percibida, felicidad y estrategias distractoras en adolescentes. Boletín de Psicología, 104, 87101.

Rodríguez, A. (2005). Felicidad subjetiva y dimensiones del autoconcepto físico. Revista de Psicodidáctica, 10 (1), 131-138

SALOVEY, y otros (eds.) (1999). Coping intelligently: emotional intelligence and the coping process. En C. R. Snyder (Eds), Coping: The psychology of what works (pp.141-164). New York: Oxford University Press.

TRINIDAD, D y JOHNSON, C. (2002). The association between emocional intelligence and early adolescent tobacco and alcohol use. Personality and Individual Differences, 32, 95-105.

Vallés, A. (2007). Inteligencia Emocional para la Convivencia Escolar. El programa PIECE. Madrid: EOS. 